\title{
Dependent coordinates in path integral measure factorization
}

\author{
S. N. Storchak \\ Institute for High Energy Physics, Protvino, \\ Moscow Region, 142284, Russia, \\ e-mail: storchak@mx.ihep.su
}

\begin{abstract}
The transformation of the path integral measure under the reduction procedure in the dynamical systems with a symmetry is considered. The investigation is carried out in the case of the Wiener-type path integrals that are used for description of the diffusion on a smooth compact Riemannian manifold with the given free isometric action of the compact semisimple unimodular Lie group. The transformation of the path integral, which factorizes the path integral measure, is based on the application of the optimal nonlinear filtering equation from the stochastic theory. The integral relation between the kernels of the original and reduced semigroup are obtained.
\end{abstract}

Keywords: constrained systems, Marsden-Weinstein reduction, path integral, stochastic analysis.

PACS: 03.65.Bz, 31.15.Kb

\section{Introduction}

The standard approach to the path integral quantization of the gauge field theories is based on the Faddeev-Popov method [1], by which a path integral over invariant variables is rewritten as a path integral over variables constrained by some gauge conditions. But in order to obtain such a representation it is necessary to separate the path integral measure on two parts 
related, correspondingly, with the gauge-invariant (independent) and the gauge-dependent (or group) degrees of freedom. However, in general it is unknown: does this separation of the path integral measure leads (or not) to a some Jacobian in the path integral measure.

In the present paper we consider the path integral quantization problem for the scalar particle which moves on a manifold with a given group action. In this problem, as in gauge field theories, an original manifold can be regarded as a total space of the principle fiber bundle. As a consequence of such a representation, we get a new way of the coordinatization of an initial manifold. The coordinate functions of a chart in the manifold atlas can be given by invariant variables related with the orbit space and by the groupvalued variables defined by the group element which "measures" the distance between the considered point in the total space of the fiber bundle and the base point which can be reached along the orbit.

The path integral quantization of our problem was considered in [2, 3] by using the definitions of the path integrals based on discrete approximations. In our papers 4, 5], we studied the quantization of this problem with the help of the path integrals in which the path integral measures were defined by the stochastic processes. It was found there, that the factorization of the path integral measure can be performed by applying the nonlinear filtering equation from the stochastic process theory.

The description of the orbit space evolution in gauge field theories are usually given by the constrained (or dependent) coordinates. It means, that the corresponding variables should meet the additional conditions (the gauges). From the local point of view, these additional conditions (given by the system of equations) define a local submanifold in the original manifold. In turns, the submanifold can be regarded as a local section of the principal fiber bundle.

Since there is a local isomorphism between the initial principle fiber bundle and the trivial principal fiber bundle which has this local submanifold as the base space [6], we can also make use these dependent coordinates as the coordinates on our principal fiber bundle.

If these local submanifolds (the local sections) are the parts of a some global submanifold (a global section) then our initial principal fiber bundle is a trivial one. And in this case, our dependent coordinates have the global meaning. But in general, there is no a global section in the principal fiber bundle. So, in a local neighborhood of the each point of the initial manifold, one should introduce its own dependent coordinates. 
After performing the local factorization of the path integral measure into the "the group measure" and the measure that are given on the local section, one should to solve the problem of the definition of a some global measure related with the set of these local measures.

Recently [7, it was found the possible solution of such a problem. In this paper it was shown a way of description of the global path integral measure in terms of the local measures. Therefore, from the principal point of view, the problem of the introduction of the dependent coordinates leads us to the consideration of the particular case of the trivial principal fiber bundle.

The representation of the orbit space path integrals as the integral over the dependent variables were studied in many papers (see, for example, [8, 9, 10]). But the path integral measure factorization questions was not considered there. In the present paper, we will investigate the behavior of the path integral measure under changing the path integral variables for the dependent ones in the Wiener-like path integrals that are used to describe the "quantum" motion of the scalar particle on a smooth compact Riemannian manifold (without boundary) on which the free effective isometric action of the compact semisimple unimodular Lie group is given.

\section{Definitions}

The objects of our consideration will be the path integrals representing the solution of the backward Kolmogorov equation given on a smooth compact Riemannian manifold $\mathcal{P}$ :

$$
\left\{\begin{array}{lr}
\left(\frac{\partial}{\partial t_{a}}+\frac{1}{2} \mu^{2} \kappa \triangle_{\mathcal{P}}\left(p_{a}\right)+\frac{1}{\mu^{2} \kappa m} V\left(p_{a}\right)\right) \psi_{t_{b}}\left(p_{a}, t_{a}\right)=0 \\
\psi_{t_{b}}\left(p_{b}, t_{b}\right)=\phi_{0}\left(p_{b}\right), & \left(t_{b}>t_{a}\right),
\end{array}\right.
$$

In this equation $\mu^{2}=\frac{\hbar}{m}, \kappa$ is a real positive parameter, $\triangle_{\mathcal{P}}\left(p_{a}\right)$ is a Laplace-Beltrami operator on manifold $\mathcal{P}$, and $V(p)$ is a group-invariant potential term. In a chart $\left(\mathcal{U}, \varphi^{A}\right)$ with the coordinate functions $Q^{A}=\varphi^{A}(p)$, the Laplace - Beltrami operator is given as

$$
\triangle_{\mathcal{P}}(Q)=G^{-1 / 2}(Q) \frac{\partial}{\partial Q^{A}} G^{A B}(Q) G^{1 / 2}(Q) \frac{\partial}{\partial Q^{B}},
$$

where the matrix $G^{A B}(Q)$ is inverse to the matrix of the Riemannian metric $G_{A B}$ given in the the coordinate basis $\left\{\frac{\partial}{\partial Q^{A}}\right\}, G=\operatorname{det}\left(G_{A B}\right)$. 
In order to get the Schrödinger equation from the equation (11) one should perform the transition to the forward Kolmogorov equation and then to put $\kappa=i$ in obtaining equation. In case of some analytical restrictions imposed on the coefficients of the the backward Kolmogorov equation, its fundamental solution satisfies also a forward equation. However, the transition from the Wiener-like path integrals to the Feynman' ones is a special problem which needs an additional investigation and does not considered in the paper.

There are different representations of the solution of the equation (11) in terms of the path integral. We will use the definition of the path integral from [1], together with the assumptions that all necessary analytical conditions for this take place in our paper. By [11, the solution of (11) can be written as follows:

$$
\begin{aligned}
\psi_{t_{b}}\left(p_{a}, t_{a}\right) & =\mathrm{E}\left[\phi_{0}\left(\eta\left(t_{b}\right)\right) \exp \left\{\frac{1}{\mu^{2} \kappa m} \int_{t_{a}}^{t_{b}} V(\eta(u)) d u\right\}\right] \\
& =\int_{\Omega_{-}} d \mu^{\eta}(\omega) \phi_{0}\left(\eta\left(t_{b}\right)\right) \exp \{\ldots\},
\end{aligned}
$$

where $\eta(t)$ is a global stochastic process on a manifold $\mathcal{P}, \mu^{\eta}$ is the path integral measure on the path space $\Omega_{-}=\left\{\omega(t): \omega\left(t_{a}\right)=0, \eta(t)=p_{a}+\omega(t)\right\}$ defined by the probability distribution of a stochastic process $\eta(t)$.

On charts $(\mathcal{U}, \varphi)$ in the atlas for the manifold $\mathcal{P}$, the global stochastic process $\eta(t)$ is defined by the local processes $\varphi(\eta)=\eta_{\varphi}(t) \equiv\left\{\eta^{A}(t)\right\}$ that are solutions of the stochastic differential equations:

$$
d \eta^{A}(t)=\frac{1}{2} \mu^{2} \kappa G^{-1 / 2} \frac{\partial}{\partial Q^{B}}\left(G^{1 / 2} G^{A B}\right) d t+\mu \sqrt{\kappa} \mathfrak{X}_{\bar{M}}^{A}(\eta(t)) d w^{\bar{M}}(t),
$$

where the matrix $\mathfrak{X}_{\bar{M}}^{A}$ is defined by the local equality $\sum_{\bar{K}=1}^{n_{P}} \mathfrak{X}_{\bar{K}}^{A} \mathfrak{X}_{\bar{K}}^{B}=G^{A B}$. Here and in what follows we denote the Euclidean indices by over-barred indices.

According to [11, the global semigroup determined by the equation (2) acts in the space of smooth and bounded functions on $\mathcal{P}$. It is defined by the limit (under the refinement of the time interval) of the superposition of the local semigroups

$$
\psi_{t_{b}}\left(p_{a}, t_{a}\right)=U\left(t_{b}, t_{a}\right) \phi_{0}\left(p_{a}\right)=\lim _{q} \tilde{U}_{\eta}\left(t_{a}, t_{1}\right) \cdot \ldots \cdot \tilde{U}_{\eta}\left(t_{n-1}, t_{b}\right) \phi_{0}\left(p_{a}\right),
$$

where, in turns, each of the local semigroup $\tilde{U}_{\eta}$ is related with the local representative of the global stochastic process $\eta$. 
One of the main advantage of the definition (41) is that it gives us an opportunity to derive the transformation properties of the path integral of (2) by studying the local semigroups $\tilde{U}_{\eta}{ }^{1}$ :

$$
\tilde{U}_{\eta}(s, t) \phi(p)=\mathrm{E}_{s, p} \phi(\eta(t)), s \leq t, \eta(s)=p,
$$

These local semigroup are also defined by the path integrals with the integration measures determined by the local representatives $\varphi^{\mathcal{P}}(\eta(t))=$ $\eta^{\varphi^{\mathcal{P}}}(t) \equiv\left\{\eta^{A}(t)\right\}$ of the global stochastic process $\eta(t)$.

\section{Principal fiber bundle coordinates}

The problem, which we consider in the paper, is related with the investigation of the reduction procedure in the dynamical systems with the symmetry. Due to the symmetry, the initial dynamical system is reduced to the system that can be described in terms of the invariant variables. The geometry of this problem is well developed [12].

A free (effective) action of the compact semisimple group Lie $\mathcal{G}$ on a smooth compact manifold $\mathcal{P}^{2}$ leads to the orbit-fibering of the manifold $\mathcal{P}$. And we can regard the manifold $\mathcal{P}$ as a total space of the principal fiber bundle $P(\mathcal{M}, \mathcal{G})$ where the orbit space $\mathcal{M}$ is a base space.

The principal bundle picture means that locally manifold $\mathcal{P}$ has the product structure: $\pi^{-1}\left(\mathcal{U}_{x}\right) \sim \mathcal{U}_{x} \times \mathcal{G}$, where $\mathcal{U}_{x}$ is an open neighborhood of the point $x=\pi(p)$ which belongs to the chart $\left(\mathcal{U}_{x}, \varphi_{x}\right)$ of the bundle $P(\mathcal{M}, \mathcal{G})$. Therefore, we can equally use the principal bundle coordinates for the coordinatization of our manifold $\mathcal{P}$. In other words, we can express an initial coordinates $Q^{A}$ of the point $p$ in terms of the principal bundle coordinates $\left(x^{i}, a^{\alpha}\right)$ $\left(i=1, \ldots, N_{\mathcal{M}}, N_{\mathcal{M}}=\operatorname{dim} \mathcal{M}, \alpha=1, \ldots, N_{\mathcal{G}}, N_{\mathcal{G}}=\operatorname{dim} \mathcal{G}, N_{\mathcal{P}}=N_{\mathcal{M}}+N_{\mathcal{G}}\right)$.

We could take the set of the functionally independent and $\mathcal{G}$-invariant functions that are solutions of the special differential equations as the invariant coordinates $x^{i}(Q)$ (the orbit space coordinates) of a point. However, in many cases finding of solutions of these differential equations becomes a very difficult problem. Hopefully, there is also an another method of the

\footnotetext{
${ }^{1}$ In the following, we omit the the potential term of the Hamiltonian operator as it is inessential for us in performing the path integral transformations. It will be recovered in final formulas.

${ }^{2}$ In our case this is an isometric action on a Riemannian manifold.
} 
orbit space coordinatization in which the necessary invariant coordinates are introduced with the help of the gauge constraints.

It is supposed, that in each sufficiently small neighborhood of the point $p$, belonging to the manifold $\mathcal{P}$, there are a set of the functions $\left\{\chi^{\alpha}(Q), \alpha=\right.$ $\left.1, \ldots, N_{\mathcal{G}}\right\}$, that can be used (by the equations $\chi^{\alpha}(Q)=0$ ) to determine a some local submanifold of the manifold $\mathcal{P}$. It is required that the submanifold should have the transversal intersection with each of the orbits. Then the coordinates on the manifold $\mathcal{P}$ can be introduced as follows.

By our assumption, we have the action of the group $\mathcal{G}$ on the manifold $\mathcal{P}$ : $\tilde{p}=p a$, or in coordinates: $\tilde{Q}^{A}=F^{A}\left(Q^{B}, a^{\alpha}\right)$, where $Q^{A}$ are the coordinates of a point $p$. We assume that it is a right action, i.e., $\left(p a_{1}\right) a_{2}=p\left(a_{1} a_{2}\right)$ :

$$
F\left(F\left(Q, a_{1}\right), a_{2}\right)=F\left(Q, \Phi\left(a_{1}, a_{2}\right)\right)
$$

where $\Phi$ is the function which determines the group multiplication law in the space of the group parameters.

The group coordinates $a^{\alpha}(Q)$ of a point $p$ are defined as a coordinates of that group element which carries the point $p$ to the local submanifold $\left\{\chi^{\alpha}(Q)=0\right\}$. These group coordinates are given by the solution of the following equation:

$$
\chi^{\alpha}\left(F^{A}\left(Q, a^{-1}(Q)\right)\right)=0 .
$$

The invariant coordinates $x^{i}(Q)$ of $p$ are the coordinates of that point of the submanifold $\left\{\chi^{\alpha}(Q)=0\right\}$ which is obtained from the point $p$ under the action of the group element with the coordinates $a^{\alpha}(Q)$. If the submanifold $\left\{\chi^{\alpha}(Q)=0\right\}$ is given parametrically: $Q^{A}=Q^{* A}\left(x^{i}\right)$, the coordinates $x^{i}(Q)$ are defined by the equation:

$$
Q^{* A}\left(x^{i}\right)=F^{A}\left(Q, a^{-1}(Q)\right) .
$$

We refer to [13] where this way of the coordinatization was considered and where the geometrical generalization of the Bogolubov coordinate transformation method from [14] was obtained.

The path integral transformation induced by replacement of coordinates $Q^{A}$ for $\left(x^{i}(Q), a^{\alpha}(Q)\right)$ and the factorization of the path integral measure was considered in [5]. In the present paper we study the same problem, but now together with the group coordinates $a^{\alpha}$ (obtained by previous method) we will use the constrained (or dependent) coordinates $Q^{* A}:\left\{\chi^{\alpha}\left(Q^{* A}\right)=0\right\}$ of the corresponding point on the local submanifold $\left\{\chi^{\alpha}=0\right\}$ of the original manifold $\mathcal{P}$. 
We will assume, that these local submanifolds form a global submanifold $\Sigma$ in the original manifold $\mathcal{P}$. Therefore, our principal fiber bundle $P(\mathcal{M}, \mathcal{G})$ is a trivial one.

Provided that the coordinates $Q^{*}$ are constrained: $\left\{\chi^{\alpha}\left(Q^{*}\right)=0\right\}$, the initial coordinates $Q^{A}$ of a point $p$ are given by the equation $Q^{A}=F^{A}\left(Q^{* A}, a^{\alpha}\right)$. Later we will see that an apparent ambiguity of a transition from $Q^{A}$ to $\left(Q^{* A}, a^{\alpha}\right)$ is compensated by the presence of the corresponding projection operators in resulting expressions.

The representation of a Riemannian metric of the manifold $\mathcal{P}$ in new coordinates $\left(Q^{* A}, a^{\alpha}\right)$ is derived from the transformation of the coordinate vector fields. It is given as follows:

$$
\begin{aligned}
\frac{\partial}{\partial Q^{B}}= & F_{B}^{C}\left(F\left(Q^{*}, a\right), a^{-1}\right) N_{C}^{A}\left(Q^{*}\right) \frac{\partial}{\partial Q^{* A}} \\
& +F_{B}^{E}\left(F\left(Q^{*}, a\right), a^{-1}\right) \chi_{E}^{\mu}\left(Q^{*}\right)\left(\Phi^{-1}\right)_{\mu}^{\beta}\left(Q^{*}\right) \bar{v}_{\beta}^{\alpha}(a) \frac{\partial}{\partial a^{\alpha}}
\end{aligned}
$$

Here $F_{B}^{C}(Q, a) \equiv \frac{\partial F^{C}}{\partial Q^{B}}(Q, a), \chi_{E}^{\mu} \equiv \frac{\partial \chi^{\mu}}{\partial Q^{E}}(Q),\left(\Phi^{-1}\right)_{\mu}^{\beta}(Q)$ - the matrix which is inverse to the Faddeev - Popov matrix:

$$
(\Phi)_{\mu}^{\beta}(Q)=K_{\mu}^{A}(Q) \frac{\partial \chi^{\beta}(Q)}{\partial Q^{A}}
$$

$\left(K_{\mu}\right.$ are the Killing vector fields for the Riemannian metric $G_{A B}(Q)$ ), the matrix $\bar{v}_{\beta}^{\alpha}(a)$ is inverse to the matrix $\bar{u}_{\beta}^{\alpha}(a)$. The $\operatorname{det} \bar{u}_{\beta}^{\alpha}(a)$ is a density of a right invariant measure given on the group $\mathcal{G}$.

Finally, $N_{C}^{A}$ is a matrix form of the projection operator $\left(N_{B}^{A} N_{C}^{B}=N_{C}^{A}\right)$ which project onto the orthogonal to the Killing vector field subspace:

$$
N_{C}^{A}(Q)=\delta_{C}^{A}-K_{\alpha}^{A}(Q)\left(\Phi^{-1}\right)_{\mu}^{\alpha}(Q) \chi_{C}^{\mu}(Q) .
$$

In (5), this projection operator is restricted to the submanifold $\left\{\chi^{\alpha}=0\right\}$ :

$$
\begin{aligned}
& N_{D}^{M}\left(Q^{*}\right) \equiv N_{D}^{M}\left(F\left(Q^{*}, e\right)\right), \\
& N_{D}^{M}\left(Q^{*}\right)=F_{D}^{B}\left(Q^{*}, a\right) N_{B}^{A}\left(F\left(Q^{*}, a\right)\right) F_{A}^{M}\left(F\left(Q^{*}, a\right), a^{-1}\right) .
\end{aligned}
$$

The formula (5) is similar to the corresponding formula from [9, 15]. On treatment of the dependent coordinate we refer, for example, to [16].

As an operator, the vector field $\frac{\partial}{\partial Q^{* A}}$ is defined by the rule:

$$
\frac{\partial}{\partial Q^{* A}} \varphi\left(Q^{*}\right)=\left.\left(P_{\perp}\right)_{A}^{D}\left(Q^{*}\right) \frac{\partial \varphi(Q)}{\partial Q^{D}}\right|_{Q=Q^{*}},
$$


where $P_{\perp}$ is a projection operator on the tangent plane to the submanifold given by the gauges:

$$
\left(P_{\perp}\right)_{B}^{A}=\delta_{B}^{A}-\chi_{B}^{\alpha}\left(\chi \chi^{\top}\right)_{\alpha}^{-1 \beta}\left(\chi^{\top}\right)_{\beta}^{A}
$$

Here $\left(\chi^{\top}\right)_{\beta}^{A}$ is a transposed matrix to the matrix $\chi_{B}^{\nu}$ :

$$
\left(\chi^{\top}\right)_{\mu}^{A}=G^{A B} \gamma_{\mu \nu} \chi_{B}^{\nu}, \quad \gamma_{\mu \nu}=K_{\mu}^{A} G_{A B} K_{\nu}^{B} .
$$

The above projection operators have the following properties:

$$
\left(P_{\perp}\right)_{B}^{\tilde{A}} N_{\tilde{A}}^{C}=\left(P_{\perp}\right)_{B}^{C}, \quad N_{B}^{\tilde{A}}\left(P_{\perp}\right)_{\tilde{A}}^{C}=N_{B}^{C}
$$

In new coordinate basis the metric $G_{A B}$ is written as a metric $\tilde{G}_{\mathcal{A B}}\left(Q^{*}, a\right)$ with the following components:

$$
\left(\begin{array}{cc}
G_{C D}\left(Q^{*}\right)\left(P_{\perp}\right)_{A}^{C}\left(P_{\perp}\right){ }_{B}^{D} & G_{C D}\left(Q^{*}\right)\left(P_{\perp}\right)_{A}^{D} K_{\mu}^{C} \bar{u}_{\alpha}^{\mu}(a) \\
G_{C D}\left(Q^{*}\right)\left(P_{\perp}\right)_{A}^{C} K_{\nu}^{D} \bar{u}_{\beta}^{\nu}(a) & \gamma_{\mu \nu}\left(Q^{*}\right) \bar{u}_{\alpha}^{\mu}(a) \bar{u}_{\beta}^{\nu}(a)
\end{array}\right),
$$

where the projection operators $P_{\perp}$ depend on $Q^{*}$, i.e., they are restricted to the submanifold, $G_{C D}\left(Q^{*}\right) \equiv G_{C D}\left(F\left(Q^{*}, e\right)\right)$ :

$$
G_{C D}\left(Q^{*}\right)=F_{C}^{M}\left(Q^{*}, a\right) F_{D}^{N}\left(Q^{*}, a\right) G_{M N}\left(F\left(Q^{*}, a\right)\right) .
$$

The pseudoinverse matrix $\tilde{G}^{\mathcal{A B}}\left(Q^{*}, a\right)$ to the matrix (6) is as follows:

$$
\left(\begin{array}{cc}
G^{E F} N_{E}^{C} N_{F}^{D} & G^{S D} N_{S}^{C} \chi_{D}^{\mu}\left(\Phi^{-1}\right)_{\mu}^{\nu} \bar{v}_{\nu}^{\sigma} \\
G^{C B} \chi_{C}^{\gamma}\left(\Phi^{-1}\right)_{\gamma}^{\beta} N_{B}^{D} \bar{v}_{\beta}^{\alpha} & G^{C B} \chi_{C}^{\gamma}\left(\Phi^{-1}\right)_{\gamma}^{\beta} \chi_{B}^{\mu}\left(\Phi^{-1}\right)_{\mu}^{\nu} \bar{v}_{\beta}^{\alpha} \bar{v}_{\nu}^{\sigma}
\end{array}\right) .
$$

In (17), $\bar{v}_{\nu}^{\sigma} \equiv \bar{v}_{\nu}^{\sigma}(a)$ and other components depend on $Q^{*}$.

The pseudoinversion of $\tilde{G}_{\mathcal{B C}}$ means that

$$
\tilde{G}^{\mathcal{A B}} \tilde{G}_{\mathcal{B C}}=\left(\begin{array}{cc}
\left(P_{\perp}\right)_{B}^{C} & 0 \\
0 & \delta_{\beta}^{\alpha}
\end{array}\right)
$$

The determinant of the matrix (6) is equal to

$$
\begin{aligned}
& \left(\operatorname{det} \tilde{G}_{\mathcal{A B}}\right)=\operatorname{det} G_{A B}\left(Q^{*}\right) \operatorname{det} \gamma_{\alpha \beta}\left(Q^{*}\right)\left(\operatorname{det} \chi \chi^{\top}\right)^{-1}\left(Q^{*}\right)\left(\operatorname{det} \bar{u}_{\nu}^{\mu}(a)\right)^{2} \\
& \quad \times\left(\operatorname{det} \Phi_{\beta}^{\alpha}\left(Q^{*}\right)\right)^{2} \operatorname{det}\left(P_{\perp}\right)_{B}^{C}\left(Q^{*}\right) .
\end{aligned}
$$

It does not vanish only on the surface $\{\chi=0\}$. On this surface $\operatorname{det}\left(P_{\perp}\right)_{B}^{C}$ is equal to unity. 


\section{Transformation of the stochastic process and the semigroup}

In result of the coordinate replacement, the local stochastic processes $\eta^{A}(t)$ on the principal fiber bundle, will get their new representations. Applying the methods of [11 to obtained local processes we can form a new global process $\zeta(t)$. It means that we have performed the transformation of the global process $\eta(t)$ to the process $\zeta(t)$.

The global process $\zeta(t)$ has two kind of the local components: $\left(Q^{* A}(t), a^{\alpha}(t)\right)$. The components $a^{\alpha}(t)$ describe the part of the stochastic evolution that originates from the stochastic evolution that was given on the group $\mathcal{G}$. The $Q^{* A}(t)$ - evolution has its origin in the stochastic evolution given on the submanifold $\Sigma$.

Although the process $Q^{* A}(t)$ is described in terms of the dependent coordinates, the transformation of the local stochastic process $\eta^{A}(t)$ for the process $\zeta^{\mathcal{A}}(t)=\left(Q^{* A}(t), a^{\alpha}(t)\right)$ is, in fact, the phase space transformation of the process $\eta^{A}(t)$. It take place because the variables $Q^{* A}$ are constrained by the condition: $\chi^{\alpha}\left(Q^{*}\right)=0$ and it is valid for the stochastic processes too.

But it is known that the phase space transformation of the stochastic processes does not change the probabilities. It means that the action of the local semigroup $\tilde{U}_{\eta}$ on a function $\varphi_{0}(p)$ is equal to the expectation of the transformed function given a $\sigma$-algebra generated by the transformed process $\zeta^{\mathcal{A}}(t)$.

On charts of the manifold $\mathcal{P}$, this transition to new coordinates can be considered as follows. The local semigroup

$$
\tilde{U}_{\eta}(s, t) \phi_{0}(p)=\mathrm{E}_{s, p} \phi_{0}(\eta(t)), \quad s \leq t, \eta(s)=p
$$

for the process $\eta(t)$ which is restricted to the chart $\left(\mathcal{V}_{p}, \varphi^{\mathcal{P}}\right)$,

$$
\varphi^{\mathcal{P}}(\eta(t))=\eta^{\varphi^{\mathcal{P}}}(t) \equiv\left\{\eta^{A}(t)\right\}
$$

can be written as

$$
\tilde{U}_{\eta}(s, t) \phi_{0}(p)=\mathrm{E}_{s, \varphi^{\mathcal{P}}(p)} \phi_{0}\left(\left(\varphi^{\mathcal{P}}\right)^{-1}\left(\eta^{\varphi^{\mathcal{P}}}(t)\right)\right), \quad \eta^{\varphi^{\mathcal{P}}}(s)=\varphi^{\mathcal{P}}(p) .
$$

The phase space transformation of the local stochastic processes

$$
\eta^{A}(t)=F^{A}\left(Q^{* B}(t), a^{\alpha}(t)\right)
$$


transforms the local semigroup $\tilde{U}_{\eta}$ :

$$
\tilde{U}_{\eta}(s, t) \phi_{0}(p)=\mathrm{E}_{s, \tilde{\varphi}^{\mathcal{P}}(p)} \phi_{0}\left(\left(\tilde{\varphi}^{\mathcal{P}}\right)^{-1}\left(\zeta^{\tilde{\varphi}^{\mathcal{P}}}(t)\right)\right)=\mathrm{E}_{s, \tilde{\varphi}^{\mathcal{P}}(p)} \tilde{\phi}_{0}\left(\zeta^{\tilde{\varphi}^{\mathcal{P}}}(t)\right),
$$

where $\left(\tilde{\varphi}^{\mathcal{P}}\right)^{-1}=\left(\varphi^{\mathcal{P}}\right)^{-1} \circ F$ and $\tilde{\phi}_{0}=\phi_{0} \circ\left(\tilde{\varphi}^{\mathcal{P}}\right)^{-1}$.

Notice, that there is a local isomorphism of the principal fiber bundle $P(\mathcal{M}, \mathcal{G})$ and the trivial principal fiber bundle $P_{\Sigma}=\Sigma \times \mathcal{G} \rightarrow \Sigma$ [6] []. From this fact it follows that we can introduce such charts in the atlas for the total space $\mathcal{P}$ of the principal fiber bundle $P(\mathcal{M}, \mathcal{G})$ that are related with the submanifold $\Sigma$.

Therefore, in our local semigroups we should take the expectation values with respect to the measures defined by the probability distribution of the local processes $\zeta^{\tilde{\varphi}^{\mathcal{P}}}(t)=\left(Q^{* A}(t), a^{\alpha}(t)\right)$. If these processes are consistent with each other on overlapping of the charts, we can define, by the method of [11], the global process and global semigroup In turns, the fact of the consistence of the local processes is verified by studying the transformations of the local stochastic differential equations that are used to define the local stochastic processes.

\section{Stochastic differential equations}

Let us consider the stochastic differential equation for the component $Q^{* A}$ of the local stochastic process $\zeta^{\mathcal{A}}(t)=\left(Q^{* A}(t), a^{\alpha}(t)\right)$. We suppose that the stochastic differential equation for this variable has the following form:

$$
d Q^{* A}(t)=b^{* A}(t) d t+c^{* A}(t) d w^{\bar{B}}(t),
$$

where we should define explicitly the drift and the diffusion coefficient.

Being subjected to the constraint condition $\chi^{\alpha}\left(Q^{*}\right)=0$, the coordinates $Q^{* A}$ are the functions of $Q^{A}$ :

$$
Q^{* A}=F^{A}\left(Q, a^{-1}(Q)\right)
$$

The stochastic variable $\eta^{A}(t)$ will have the same dependence on the stochastic variable $Q^{* A}(t)$.

Applying the Itô differentiation formula to the stochastic variable $Q^{* A}(t)$ we rewrite the left-hand side of the equation (8) as follows:

$$
d Q^{* A}(t)=\frac{\partial Q^{* A}}{\partial Q^{E}} d \eta^{E}(t)+\frac{1}{2} \frac{\partial^{2} Q^{* A}}{\partial Q^{E} \partial Q^{C}}<d \eta^{E}(t) d \eta^{C}(t)>
$$


Then, putting an expression of the stochastic differential $d \eta^{A}(t)$ from (3) into the right-hand side of (9), we obtain:

$$
\begin{aligned}
& d Q^{* A}(t)=\frac{\partial Q^{* A}}{\partial Q^{E}}\left(-\frac{1}{2} \mu^{2} \kappa G^{P B}(\eta(t)) \Gamma_{P B}^{E}(\eta(t)) d t\right. \\
& \left.+\mu \sqrt{\kappa} \mathfrak{X}_{\bar{M}}^{E}(\eta(t)) d w^{\bar{M}}(t)\right)+\frac{1}{2} \frac{\partial^{2} Q^{* A}}{\partial Q^{E} \partial Q^{C}}<d \eta^{E}(t) d \eta^{C}(t)>
\end{aligned}
$$

where $\Gamma_{P B}^{E}$ are the Christoffel coefficients for the Riemannian metric $G_{A B}$. In order to express the stochastic variable $\eta^{A}(t)$ in the last equation in terms of $Q^{* A}(t)$ and $a^{\alpha}(t)$ we make use the equation $\eta^{A}(t)=F^{A}\left(Q^{* B}(t), a^{\alpha}(t)\right)$.

After such a transformation we find that the coefficient which stands at the differential $d t$ in the obtained expression will be the drift of the equation (8). And, correspondently, the term at the stochastic differential $d w(t)$ will be the diffusion coefficient. As a result we obtain the following equation for $Q^{*}(t)$ :

$$
\begin{aligned}
& d Q^{* A}(t)=\frac{1}{2} \mu^{2} \kappa\left[N _ { C } ^ { A } N _ { M } ^ { R } \left(G\left(Q^{*}(t)\right)^{-1 / 2} \frac{\partial}{\partial Q^{* R}}\left(G\left(Q^{*}(t)\right)^{1 / 2} G^{C M}\left(Q^{*}(t)\right)\right)\right.\right. \\
& +N_{C L}^{A} G^{C L}-G^{P C} N_{C}^{K} K_{\mu M}^{M}\left(\Phi^{-1}\right)_{\nu}^{\mu} \chi_{P}^{\nu}+G^{P C} N_{C}^{A} K_{\mu P}^{E}\left(\Phi^{-1}\right)_{\nu}^{\mu} \chi_{E}^{\nu} \\
& \left.+G^{P B} N_{C}^{A} K_{\mu B}^{C}\left(\Phi^{-1}\right)_{\nu}^{\mu} \chi_{P}^{\nu}\right] d t+\mu \sqrt{\kappa} N_{C}^{A} \tilde{\mathfrak{X}}_{\bar{M}}^{C}\left(Q^{*}(t)\right) d w^{\bar{M}}(t) .
\end{aligned}
$$

In this equation all variables depend on $Q^{*}(t)$ and by additional lower indices we denote the corresponding derivatives. (For example, $N_{C L}^{A}\left(Q^{*}\right) \equiv$ $\left.\left.\frac{\partial}{\partial Q^{L}} N_{C}^{A}(Q)\right|_{Q=Q^{*}}\right)$

Also, $\tilde{\mathfrak{X}}$ from (11) is defined by $\sum_{\bar{K}=1}^{n_{P}} \tilde{\mathfrak{X}}_{\bar{K}}^{A}\left(Q^{*}\right) \tilde{\mathfrak{X}}_{\bar{K}}^{B}\left(Q^{*}\right)=G^{A B}\left(Q^{*}\right)$.

The drift term of equation (11) has another representation, which is related with the geometrical objects that are specific for the considered problem. In order to the derive this representation we make use the following expansion of the operator Laplace - Beltrami from [3]:

$$
\frac{1}{2} \triangle_{\mathcal{P}}=\frac{1}{2}\left({ }^{\perp} G^{A B} \nabla_{A} \nabla_{B}-K_{\mu}^{A} \gamma^{\mu \nu}\left(\nabla_{A} K_{\nu}^{B}\right) \nabla_{B}+K_{\alpha}^{A} \gamma^{\alpha \beta} \nabla_{B} K_{\beta}^{B} \nabla_{B}\right),
$$

where ${ }^{\perp} G^{A B}=G^{A B}-K_{\alpha}^{A} \gamma^{\alpha \beta} K_{\beta}^{B}$, and $\nabla_{A}$ is the symbol of the covariant derivative which is obtained from the Christoffel coefficients for the original Riemannian metric $G_{A B}(Q)$.

If we replace $Q$ for $\left(Q^{*}, a\right)$ in (12), then it can be shown that the drift in (11) is a sum of two terms: $b_{I}^{A}\left(Q^{*}\right)$ and $b_{I I}^{A}\left(Q^{*}\right)$. They are equal to those 
coefficients at the first partial derivatives over $Q^{*}$ that come from the first and the second terms of the right-hand side of the equation (12) after changing the variables. The third term of expansion of the laplacian in (12) does not give the contribution to the terms with partial derivatives over $Q^{*}$.

Performing necessary evaluations, we find that $b_{I I}^{A}\left(Q^{*}\right)$ is the projection of the mean curvature vector of the orbit

$$
j^{D}(Q) \frac{\partial}{\partial Q^{D}}=\frac{1}{2} \Pi_{A}^{D}(Q) \gamma^{\alpha \beta}(Q)\left[\nabla_{K_{\alpha}(Q)} K_{\beta}(Q)\right]^{A} \frac{\partial}{\partial Q^{D}}
$$

on the submanifold $\left\{\chi^{\alpha}=0\right\}$. The projection is given with the help of the transformed metric $\widetilde{G}^{\mathcal{A B}}$ as follows:

$$
\tilde{G}^{S L} \tilde{G}\left(j^{D} \frac{\partial}{\partial Q^{D}}, \frac{\partial}{\partial Q^{* S}}\right) \frac{\partial}{\partial Q^{* L}},
$$

where before taking the projection one should change the variables $Q$ in $j^{D} \frac{\partial}{\partial Q^{D}}$ for $Q^{*}$ and $a$. The projection operator $\Pi_{A}^{D}=\delta_{A}^{D}-K_{\mu}^{D} \gamma^{\mu \nu} K_{\nu}^{C} G_{C A}$ extracts the direction which is normal to the orbit: $\Pi_{A}^{D} K_{\alpha}^{A}=0$.

As a result of the projection we get the following expression for the $b_{I I}^{A}\left(Q^{*}\right)$ :

$$
b_{I I}^{A}\left(Q^{*}\right)=\frac{1}{2} G^{E U} N_{E}^{A} N_{U}^{D}\left[\gamma^{\alpha \beta} G_{C D}\left(\tilde{\nabla}_{K_{\alpha}} K_{\beta}\right)^{C}\right],
$$

in which all the values from the right-hand side depend on $Q^{*}$ and by $\left(\tilde{\nabla}_{K_{\alpha}} K_{\beta}\right)^{C}\left(Q^{*}\right)$ we denote

$$
\left.K_{\alpha}^{A}\left(Q^{*}\right) \frac{\partial}{\partial Q^{A}} K_{\beta}^{C}(Q)\right|_{Q=Q^{*}}+K_{\alpha}^{A}\left(Q^{*}\right) K_{\beta}^{B}\left(Q^{*}\right) \tilde{\Gamma}_{A B}^{C}\left(Q^{*}\right)
$$

where

$$
\begin{aligned}
& \tilde{\Gamma}_{A B}^{C}\left(Q^{*}\right)= \\
& \quad \frac{1}{2} G^{C E}\left(Q^{*}\right)\left(\frac{\partial}{\partial Q^{* A}} G_{E B}\left(Q^{*}\right)+\frac{\partial}{\partial Q^{* B}} G_{E A}\left(Q^{*}\right)-\frac{\partial}{\partial Q^{* E}} G_{A B}\left(Q^{*}\right)\right) .
\end{aligned}
$$

The relation of $b_{I}^{A}\left(Q^{*}\right)$, which comes from the first term of an expansion of the laplacian in (12), with the geometry of the problem can be found as follows.

In the local picture, the projection onto the orbit space $\mathcal{M}$, which is locally isomorphic to $\Sigma$, is realized by replacement of the coordinates: $Q^{A}=$ 
$Q^{* A}(x)$. Under this replacement the first term in the right-hand side of equation (12) transforms into the Laplace - Beltrami operator of the manifold $\left(\mathcal{M}, h_{i j}\right)$ with the induced metric

$$
h_{i j}(x)=Q_{i}^{* A}(x) G_{A B}^{H}\left(Q^{*}(x)\right) Q_{j}^{* B}(x) .
$$

We can also regard the orbit space as a submanifold of the (Riemannian) manifold $\left(\mathcal{P}, G_{A B}^{H}(Q)\right)$ with the degenerate metric $G_{A B}^{H}$.

The orbit space diffusion is described locally by the following stochastic differential equation:

$$
d x^{i}(t)=-\frac{1}{2} \mu^{2} \kappa h^{k l}(x(t)) \Gamma_{k l}^{i}(x(t)) d t+\mu \sqrt{\kappa} X_{\bar{m}}^{i}(x(t)) d w^{\bar{m}}(t),
$$

in which the Christoffel coefficients correspond to the metric $h_{i j}(x)$.

But, besides the standard description of this diffusion in terms of the internal variables that are given on the submanifold, there is a description of the same diffusion with the help of the stochastic differential equation defined in terms of the variables of the external manifold. In [17], it was considered the particular case of such a description when it was used the Euclidean space as an external manifold. It is not difficult to find a similar discription for a general case (see Appendix A).

To derive the corresponding stochastic differential equation of our problem one should repeate the evaluation that was done in Appendix A.

We remark, that in our case the metric $G_{A B}^{H}$ of an external manifold is degenerated one. Therefore, instead of the relation (A.4) from Appendix A we will have

$$
h^{k l}(x) \Gamma_{k l}^{i}=G_{A B}^{H}\left(Q_{k l}^{* A} h^{k l}+{ }^{H} \Gamma_{C D}^{A} Q_{k}^{* C} Q_{l}^{* D} h^{k l}\right) h^{i m} Q_{m}^{* B},
$$

where the multiplication $G_{A B}^{H}\left(Q^{*}(x)\right){ }^{H} \Gamma_{C D}^{B}\left(Q^{*}(x)\right)$ is defined as

$$
G_{A B}^{H}{ }^{H} \Gamma_{C D}^{B}=\frac{1}{2}\left(G_{A C, D}^{H}+G_{A D, C}^{H}-G_{C D, A}^{H}\right) .
$$

In (13), by the derivatives we mean the following: $\left.G_{A C, D}^{H} \equiv \frac{\partial G_{A C}^{H}(Q)}{\partial Q^{D}}\right|_{Q=Q^{*}(x)}$.

Repeating all the steps that was done in the Application we can show that in result of the replacement of the variables the drift of the obtained stochastic differential equation coincides with the coefficient which stands at the first partial derivative over $Q^{*}$ in the term that arise from the first term 
of the expansion of the laplacian in (12). Hence, this drift coincides also with $b_{I}$. The last means that $b_{I}$ should be related with the geometric values that characterize the orbit space.

Performing the aforementioned transformations we get the following expression for $b_{I}$ :

$$
b_{I}^{A}\left(Q^{*}(x)\right)=-\frac{1}{2} G^{E M}\left(Q^{*}(x)\right) N_{E}^{C}\left(Q^{*}(x)\right) N_{M}^{B}\left(Q^{*}(x)\right)^{H} \Gamma_{C B}^{A}\left(Q^{*}(x)\right)+j_{I}^{A},
$$

where $j_{I}$ is the mean curvature vector of the orbit space. It can be evaluated as follows:

$$
j_{I}^{A}=\frac{1}{2}\left(\delta_{B}^{A}-N_{B}^{A}\left(Q^{*}(x)\right)\right) h^{i j}(x)\left[Q_{i j}^{* B}+Q_{i}^{* P} Q_{j}^{* L}{ }_{j}^{H} \Gamma_{P L}^{B}\left(Q^{*}(x)\right)\right] .
$$

But, as a function, the mean curvature is given on a submanifold. So, similarly to that as was done in the Application A, we can redefine the stochastic variable $Q^{*}(x(t))$ for a new stochastic variable $Q^{*}(t)$. (We denote a new stochastic variable by the same latter.)

Notice, that from equation (13) the Christoffel symbols ${ }^{H} \Gamma_{C D}^{B}$ are defined up to the terms $T_{C D}^{B}$ that are satisfied to $G_{A B}^{H} T_{C D}^{B}=0$. However, this ambiguity is not essential, since $b_{I}$ can be also presented as

$$
b_{I}^{A}=-\frac{1}{2} N_{E}^{A H} \Gamma_{C D}^{E} N_{K}^{C} N_{U}^{D} G^{K U}+\frac{1}{2} N_{L M}^{A} N_{K}^{L} N_{U}^{M} G^{K U} .
$$

Therefore, in result of the transformation of equation (8) we get the following stochastic differential equation:

$$
d Q^{* A}(t)=\mu^{2} \kappa\left(-\frac{1}{2} G^{E M} N_{E}^{C} N_{M}^{B}{ }^{H} \Gamma_{C B}^{A}+j_{I}^{A}+j_{I I}^{A}\right) d t+\mu \sqrt{\kappa} N_{C}^{A} \tilde{\mathfrak{X}}_{\bar{M}}^{C} d w^{\bar{M}},
$$

where all the values from the right-hand side now depend on $Q^{*}(t)$ and we have introduced a new notation for $b_{I I}^{A}$. In new notation it is denoted by $j_{I I}^{A}$.

As for the stochastic differential equation for the group variable $a^{\alpha}(t)$, it can be obtained by the same method as it was done for the variable $Q^{* A}(t)$. It can be found that this equation is the following:

$$
\begin{aligned}
d a^{\alpha} & =-\frac{1}{2} \mu^{2} \kappa\left[G^{R S} \tilde{\Gamma}_{R S}^{B}\left(Q^{*}\right) \Lambda_{B}^{\beta} \bar{v}_{\beta}^{\alpha}+G^{R P} \Lambda_{R}^{\sigma} \Lambda_{B}^{\beta} K_{\sigma P}^{B} \bar{v}_{\beta}^{\alpha}\right. \\
& \left.-G^{C A} N_{C}^{M} \frac{\partial}{\partial Q^{* M}}\left(\Lambda_{A}^{\beta}\right) \bar{v}_{\beta}^{\alpha}-G^{M B} \Lambda_{M}^{\epsilon} \Lambda_{B}^{\beta} \bar{v}_{\epsilon}^{\nu} \frac{\partial}{\partial a^{\nu}}\left(\bar{v}_{\beta}^{\alpha}\right)\right] d t \\
& +\mu \sqrt{\kappa} \bar{v}_{\beta}^{\alpha} \Lambda_{B}^{\beta} \tilde{\mathfrak{X}}_{\bar{M}}^{B} d w^{\bar{M}},
\end{aligned}
$$


where $\bar{v} \equiv \bar{v}(a)$ and other coefficients depend on $Q^{*}$. Also, we have introduced a new notation:

$$
\Lambda_{B}^{\alpha}=\left(\Phi^{-1}\right)_{\mu}^{\alpha} \chi_{B}^{\mu}
$$

In (15), the Christoffel symbols $\tilde{\Gamma}_{R S}^{B}\left(Q^{*}\right)$ are obtained from $\Gamma_{B C}^{A}(Q)$, if in its definition we rewrite the derivatives by the formula (5).

Therefore, the stochastic process $\zeta(t)$ is given locally by the solution of the stochastic differential equations (14) and (15). On charts of the manifold the set of the solutions of these equations determine the local stochastic evolution families of mappings of the manifold $\mathcal{P}$.

As in 11, with these local families it is possible to define the global stochastic process $\zeta(t)$ which consist of two components related with the stochastic evolution on the submanifold $\Sigma$ (the gauge surface) and with the stochastic evolution on the orbit of the principal fiber bundle.

The performed transformation of the stochastic process $\eta(t)$ results to the corresponding transformation of the global semigroup (4). Now our semigroup is determined by the superposition of the local semigroups $\tilde{U}_{\zeta^{\varphi^{P}}}$ :

$$
\psi_{t_{b}}\left(p_{a}, t_{a}\right)=\lim _{q} \tilde{U}_{\zeta^{\varphi} P}\left(t_{a}, t_{1}\right) \cdot \ldots \cdot \tilde{U}_{\zeta^{\varphi}}\left(t_{n-1}, t_{b}\right) \tilde{\phi}_{0}\left(Q_{a}^{*}, \theta_{a}\right),
$$

where

$$
\tilde{U}_{\zeta^{\varphi^{P}}}(s, t) \tilde{\phi}_{0}\left(Q_{0}^{*}, \theta_{0}\right)=\mathrm{E}_{s,\left(Q_{0}^{*}, \theta_{0}\right)} \tilde{\phi}_{0}\left(Q^{*}(t), a(t)\right), \quad Q^{*}(s)=Q_{0}^{*}, \quad a(s)=\theta_{0} .
$$

We will write this global semigroup in the following symbolical form:

$$
\psi_{t_{b}}\left(p_{a}, t_{a}\right)=\mathrm{E}\left[\tilde{\phi}_{0}\left(\xi_{\Sigma}\left(t_{b}\right), a\left(t_{b}\right)\right) \exp \left\{\frac{1}{\mu^{2} \kappa m} \int_{t_{a}}^{t_{b}} \tilde{V}\left(\xi_{\Sigma}(u)\right) d u\right\}\right],
$$

where $\xi_{\Sigma}\left(t_{a}\right)=Q_{a}^{*}, a\left(t_{a}\right)=\theta_{a}, \varphi^{P}\left(p_{a}\right)=\left(Q_{a}^{*}, \theta_{a}\right)$ and we have taken into account the omitted potential term.

From (14) and (15) it follows that the coordinate representation of the differential generator of the semigroup related with the stochastic process $\zeta(t)$ is given by

$$
\begin{aligned}
& \frac{1}{2} \mu^{2} \kappa\left(G^{C D} N_{C}^{A} N_{D}^{B} \frac{\partial^{2}}{\partial Q^{* A} \partial Q^{* B}}-G^{C D} N_{C}^{A} N_{D}^{B}{ }^{H} \Gamma_{A B}^{E} \frac{\partial}{\partial Q^{* E}}+j_{I}^{A} \frac{\partial}{\partial Q^{* A}}\right. \\
& +j_{I I}^{A} \frac{\partial}{\partial Q^{* A}}+G^{A B} \Lambda_{A}^{\alpha} \Lambda_{B}^{\beta} \bar{L}_{\alpha} \bar{L}_{\beta}-G^{R S} \tilde{\Gamma}_{R S}^{B} \Lambda_{B}^{\alpha} \bar{L}_{\alpha}-G^{R P} \Lambda_{R}^{\sigma} \Lambda_{B}^{\alpha} K_{\sigma P}^{B} \bar{L}_{\alpha} \\
& \left.+G^{C A} N_{C}^{M} \frac{\partial}{\partial Q^{* M}}\left(\Lambda_{A}^{\alpha}\right) \bar{L}_{\alpha}+2 G^{B C} N_{C}^{A} \Lambda_{B}^{\alpha} \bar{L}_{\alpha} \frac{\partial}{\partial Q^{* A}}\right)+\frac{1}{\mu^{2} \kappa m} \tilde{V} .
\end{aligned}
$$


Here, all the values, except for $\bar{L}$, depend on $Q^{*}$.

\section{Factorization of the path integral measure}

In 4, 5, a new method of factorization of the path integral measure was proposed. Here, we will apply it to our case of constrained (by the equation $\chi^{\alpha}\left(Q^{*}\right)=0$ ) integration variables.

The main idea of [4] was in exploiting the stochastic differential equation from the nonlinear filtering theory [18, 19]. This equation describes the evolution of the conditional mathematical expectation of the signal process ( the process $a(t)$ in our case) with respect to the $\sigma$-algebra generated by an observable process (the stochastic process $Q^{*}(t)$ ).

In order to make use of this equation we transform each local semigroup $\tilde{U}_{\zeta^{\varphi^{P}}}$ from (16) as follows:

$$
\tilde{U}_{\zeta^{P}}(s, t) \tilde{\phi}\left(Q_{0}^{*}, \theta_{0}\right)=\mathrm{E}\left[\mathrm{E}\left[\tilde{\phi}\left(Q^{*}(t), a(t)\right) \mid\left(\mathcal{F}_{Q^{*}}\right)_{s}^{t}\right]\right] .
$$

The above transformation is based on the properties of the conditional expectation of the Markov processes. Such a path integral transformation can be also regarded as an analog of the transition from the multiple integrals to the repeated ones in the ordinary integration. tion

Being the integrand of the "repeated" integral, the conditional expecta-

$$
\hat{\widetilde{\phi}}\left(Q^{*}(t)\right) \equiv \mathrm{E}\left[\tilde{\phi}\left(Q^{*}(t), a(t)\right) \mid\left(\mathcal{F}_{Q^{*}}\right)_{s}^{t}\right],
$$

should satisfy the nonlinear filtering equation. With account of our stochastic differential equations (14) and (15), we get that it will be as follows:

$$
\begin{aligned}
& d \hat{\tilde{\phi}}=-\frac{1}{2} \mu^{2} \kappa\left(G^{R S} \tilde{\Gamma}_{R S}^{B} \Lambda_{B}^{\beta}+G^{R P} \Lambda_{R}^{\sigma} \Lambda_{B}^{\beta} K_{P \sigma}^{B}-G^{C A} N_{C}^{M} \frac{\partial}{\partial Q^{* M}}\left(\Lambda_{A}^{\beta}\right)\right) \\
& \times \mathrm{E}\left[\bar{L}_{\beta} \tilde{\phi} \mid\left(\mathcal{F}_{Q^{*}}\right)_{s}^{t}\right] d t+\frac{1}{2} \mu^{2} \kappa G^{C B} \Lambda_{C}^{\nu} \Lambda_{B}^{\kappa} \mathrm{E}\left[\bar{L}_{\nu} \bar{L}_{\kappa} \tilde{\phi} \mid\left(\mathcal{F}_{Q^{*}}\right)_{s}^{t}\right] d t \\
& +\mu \sqrt{\kappa} \Lambda_{C}^{\beta} \Pi_{K}^{C} \tilde{\mathfrak{X}}_{\bar{M}}^{K} \mathrm{E}\left[\bar{L}_{\beta} \tilde{\phi} \mid\left(\mathcal{F}_{Q^{*}}\right)_{s}^{t}\right] d w^{\bar{M}}
\end{aligned}
$$

Further transformation of the equation (18) consists in separation of the space variables from the group ones. It can be done by applying the Peter 
- Weyl theorem to the function $\widetilde{\phi}$ considered as the function on a group $\mathcal{G}$. An expansion of this function in a series yields

$$
\tilde{\phi}\left(Q^{*}, a\right)=\sum_{\lambda, p, q} c_{p q}^{\lambda}\left(Q^{*}\right) D_{p q}^{\lambda}(a),
$$

where $D_{p q}^{\lambda}(a)$ are the matrix elements of an irreducible representation $T^{\lambda}$ of the group $\mathcal{G}: \sum_{q} D_{p q}^{\lambda}(a) D_{q n}^{\lambda}(b)=D_{p n}^{\lambda}(a b)$.

By the properties of the conditional mathematical expectations we have

$$
\begin{aligned}
\left.\mathrm{E}\left[\tilde{\phi}\left(Q^{*}(t), a(t)\right) \mid(\mathcal{F})_{Q^{*}}\right)_{s}^{t}\right] & =\sum_{\lambda, p, q} c_{p q}^{\lambda}\left(Q^{*}(t)\right) \mathrm{E}\left[D_{p q}^{\lambda}(a(t)) \mid\left(\mathcal{F}_{Q^{*}}\right)_{s}^{t}\right] \\
& \equiv \sum_{\lambda, p, q} c_{p q}^{\lambda}\left(Q^{*}(t)\right) \hat{D}_{p q}^{\lambda}\left(Q^{*}(t)\right)
\end{aligned}
$$

where

$$
c_{p q}^{\lambda}\left(Q^{*}(t)\right)=d^{\lambda} \int_{\mathcal{G}} \tilde{\varphi}\left(Q^{*}(t), \theta\right) \bar{D}_{p q}^{\lambda}(\theta) d \mu(\theta)
$$

( $d^{\lambda}$ is a dimension of an irreducible representation and $d \mu(\theta)$ is a normalized $\left(\int_{\mathcal{G}} d \mu(\theta)=1\right)$ invariant Haar measure on a group $\left.\mathcal{G}\right)$.

Then, in a similar manner as it was done in [4, 5] one can derive the stochastic differential equation for the conditional expectation $\hat{D}_{p q}^{\lambda}$ :

$$
\begin{aligned}
& d \hat{D}_{p q}^{\lambda}\left(Q^{*}(t)\right)= \\
& -\frac{1}{2} \mu^{2} \kappa\left\{\left[G^{R S} \tilde{\Gamma}_{R S}^{B} \Lambda_{B}^{\mu}+G^{R P} \Lambda_{R}^{\sigma} \Lambda_{B}^{\mu} K_{P \sigma}^{B}-G^{C A} N_{C}^{M} \frac{\partial}{\partial Q^{* M}}\left(\Lambda_{A}^{\beta}\right)\right]\right. \\
& \left.\times\left(J_{\beta}\right)_{p q^{\prime}}^{\lambda} \hat{D}_{q^{\prime} q}^{\lambda}\left(Q^{*}(t)\right)-G^{C B} \Lambda_{C}^{\alpha} \Lambda_{B}^{\nu}\left(J_{\alpha}\right)_{p q^{\prime}}^{\lambda}\left(J_{\nu}\right)_{q^{\prime} q^{\prime \prime}}^{\lambda} \hat{D}_{q^{\prime \prime} q}^{\lambda}\left(Q^{*}(t)\right)\right\} d t \\
& +\mu \sqrt{\kappa} \Lambda_{C}^{\nu} \Pi_{K}^{C}\left(J_{\nu}\right)_{p q^{\prime}}^{\lambda} \hat{D}_{q^{\prime} q}^{\lambda}\left(Q^{*}(t)\right) \tilde{\mathfrak{X}}_{\bar{M}}^{K}\left(Q^{*}(t)\right) d w^{\bar{M}}(t)
\end{aligned}
$$

in which $\left.\left(J_{\mu}\right)_{p q}^{\lambda} \equiv\left(\frac{\partial D_{p q}^{\lambda}(a)}{\partial a^{\mu}}\right)\right|_{a=e}$ are the infinitesimal generators of the representation $D^{\lambda}(a)$ :

$$
\bar{L}_{\mu} D_{p q}^{\lambda}(a)=\sum_{q^{\prime}}\left(J_{\mu}\right)_{p q^{\prime}}^{\lambda} D_{q^{\prime} q}^{\lambda}(a)
$$

Notice, that in general, the conditional expectation $\hat{D}_{p q}^{\lambda}\left(Q^{*}(t)\right)$ depend also on the initial points $Q_{0}^{*}=Q^{*}(s)$ and $\theta_{0}^{\alpha}=a^{\alpha}(s)$ besides the of the stochastic processes $Q^{*}(t)$. 
The solution of the linear matrix stochastic differential equation (19) can be written [20] as:

$$
\hat{D}_{p q}^{\lambda}\left(Q^{*}(t)\right)=(\overleftarrow{\exp })_{p n}^{\lambda}\left(Q^{*}(t), t, s\right) \mathrm{E}\left[D_{n q}^{\lambda}(a(s)) \mid\left(\mathcal{F}_{Q^{*}}\right)_{s}^{t}\right]
$$

where

$$
\begin{aligned}
& (\overleftarrow{\exp })_{p n}^{\lambda}\left(Q^{*}(t), t, s\right)=\overleftarrow{\exp } \int_{s}^{t}\left\{\frac { 1 } { 2 } \mu ^ { 2 } \kappa \left[\bar{\gamma}^{\sigma \nu}\left(Q^{*}(u)\right)\left(J_{\sigma}\right)_{p r}^{\lambda}\left(J_{\nu}\right)_{r n}^{\lambda}\right.\right. \\
& \left.-\left(G^{R S} \tilde{\Gamma}_{R S}^{B} \Lambda_{B}^{\beta}+G^{R P} \Lambda_{R}^{\sigma} \Lambda_{B}^{\beta} K_{P \sigma}^{B}-G^{C A} N_{C}^{M} \frac{\partial}{\partial Q^{* M}}\left(\Lambda_{A}^{\beta}\right)\right)\left(J_{\beta}\right)_{p n}^{\lambda}\right] d u \\
& \left.+\mu \sqrt{\kappa} \Lambda_{C}^{\beta}\left(J_{\beta}\right)_{p n}^{\lambda} \Pi_{K}^{C} \tilde{\mathfrak{X}}_{\bar{M}}^{K} d w^{\bar{M}}\right\}
\end{aligned}
$$

is a multiplicative stochastic integral. This integral is a limit of the sequence of time-ordered multipliers that have been obtained as a result of breaking of a time interval $[s, t]$. The time order of these multipliers is given by the direction of the arrow aimed to the multipliers at greater times.

With account of the representation for $\hat{D}_{p q}^{\lambda}$ obtained in (20) and (21) we rewrite our local semigroup (17) as follows:

$$
\tilde{U}_{\zeta^{\varphi^{P}}}(s, t) \tilde{\phi}\left(Q_{0}^{*}, \theta_{0}\right)=\sum_{\lambda, p, q, q^{\prime}} \mathrm{E}\left[c_{p q}^{\lambda}\left(Q^{*}(t)\right)(\overline{\exp })_{p q^{\prime}}^{\lambda}\left(Q^{*}(t), t, s\right)\right] D_{q^{\prime} q}^{\lambda}\left(\theta_{0}\right)
$$

where we have taken into account that

$$
\mathrm{E}\left[D_{n q}^{\lambda}(a(s)) \mid\left(\mathcal{F}_{Q^{*}}\right)_{s}^{t}\right]=D_{n q}^{\lambda}(a(s))=D_{n q}^{\lambda}\left(\theta_{0}\right) .
$$

In order to obtain the global semigroup by the methods of [11] one should break the time interval $\left[t_{a}, t_{b}\right]$ and should take the superposition of the local semigroups that are similar to (22). Then, the global semigroup for the global process is obtained as a limit (under the refinement of the subdivision of the time interval) of the superposition of these local semigroups. The relation between the global semigroup obtained in result of the limiting procedure can be written simbolically in the following form:

$$
\psi_{t_{b}}\left(p_{a}, t_{a}\right)=\sum_{\lambda, p, q, q^{\prime}} \mathrm{E}\left[c_{p q}^{\lambda}\left(\xi_{\Sigma}\left(t_{b}\right)\right)(\overleftarrow{\exp })_{p q^{\prime}}^{\lambda}\left(\xi_{\Sigma}(t), t_{b}, t_{a}\right)\right] D_{q^{\prime} q}^{\lambda}\left(\theta_{a}\right)
$$

$\left(\xi_{\Sigma}\left(t_{a}\right)=\left.\pi\right|_{\Sigma} \circ p_{a}\right)$

where $\xi_{\Sigma}(t)$ is a global stochastic process defined on the submanifold $\Sigma$. This process is described locally by the equations (14). 
Thus, our initial original path integral has been rewritten as the sum of the matrix semigroups (the path integrals) that are given on the submanifold $\Sigma$. The differential generator (the Hamiltonian operator) of these matrix semigroups is

$$
\begin{aligned}
& \frac{1}{2} \mu^{2} \kappa\left\{\left[G^{C D} N_{C}^{A} N_{D}^{B} \frac{\partial^{2}}{\partial Q^{* A} \partial Q^{* B}}-G^{C D} N_{C}^{E} N_{D}^{M}{ }^{H} \Gamma_{E M}^{A} \frac{\partial}{\partial Q^{* A}}\right.\right. \\
& \left.+\left(j_{I}^{A}+j_{I I}^{A}\right) \frac{\partial}{\partial Q^{* A}}\right]\left(I^{\lambda}\right)_{p q}+2 N_{C}^{A} G^{C P} \Lambda_{P}^{\alpha}\left(J_{\alpha}\right)_{p q}^{\lambda} \frac{\partial}{\partial Q^{* A}} \\
& -\left(G^{R S} \tilde{\Gamma}_{R S}^{B} \Lambda_{B}^{\alpha}+G^{R P} \Lambda_{R}^{\sigma} \Lambda_{B}^{\alpha} K_{P \sigma}^{B}-G^{C A} N_{C}^{M} \frac{\partial}{\partial Q^{* M}}\left(\Lambda_{A}^{\alpha}\right)\right)\left(J_{\alpha}\right)_{p q}^{\lambda} \\
& \left.+G^{S B} \Lambda_{B}^{\alpha} \Lambda_{S}^{\sigma}\left(J_{\alpha}\right)_{p q^{\prime}}^{\lambda}\left(J_{\sigma}\right)_{q^{\prime} q}^{\lambda}\right\}
\end{aligned}
$$

where $\left(I^{\lambda}\right)_{p q}$ is a unity matrix.

The operator acts in the space of the section $\Gamma\left(\Sigma, V^{*}\right)$ of the covector fiber bundle, which is associated with the trivial principal fiber bundle $\pi$ : $\Sigma \times \mathcal{G} \rightarrow \Sigma$. The scalar product in this space of the sections is defined as follows:

$$
\left(\psi_{n}, \psi_{m}\right)=\int_{\Sigma}\left\langle\psi_{n}, \psi_{m}\right\rangle_{V_{\lambda}^{*}} \frac{\operatorname{det} \Phi_{\beta}^{\alpha}}{\operatorname{det}^{1 / 2}\left(\chi_{A}^{\mu} G^{A B} \chi_{B}^{\nu}\right)} d v_{\Sigma},
$$

where $d v_{\Sigma}$ is the Riemannian volume element of $\Sigma$.

An integration measure of the scalar product of the formula (25) has been obtained from the Riemanian volume element of the manifold $\mathcal{P}$, in which we have changed the variables $Q^{A}$ for $\left(Q^{* i}, a^{\alpha}\right)$. Also, we have used an equality:

$$
\begin{aligned}
& \operatorname{det} G_{A B}\left(Q^{* i}, Q^{* \alpha}\left(Q^{* i}\right), a^{\mu}\right)= \\
& \quad \operatorname{det}\left(\left(G_{\Sigma}\right)_{A B}\right) \operatorname{det}^{-1}\left(\left(G^{B C} \chi_{B}^{\nu} \chi_{C}^{\mu}\right) \Phi_{\mu}^{-1 \alpha} \Phi_{\nu}^{-1 \beta} \bar{v}_{\alpha}^{\sigma} \bar{v}_{\beta}^{\rho}\right) .
\end{aligned}
$$

Notice, that the metric $\left(G_{\Sigma}\right)_{A B}\left(Q^{* i}, Q^{* \alpha}\left(Q^{* i}\right)\right)$ is a restriction of the metric $\left(P_{\perp}\right)_{A}^{C}\left(Q^{*}\right) G_{C D}\left(Q^{*}\right)\left(P_{\perp}\right)_{B}^{D}\left(Q^{*}\right)$ to the surface $\Sigma$.

Performing the transformation of the measure in the integral (25) , we can also to present the scalar product as

$$
\int\left\langle\psi_{n}, \psi_{m}\right\rangle_{V_{\lambda}^{*}} \operatorname{det} \Phi_{\beta}^{\alpha} \prod_{\alpha=1}^{N_{\mathcal{G}}} \delta\left(\chi^{\alpha}\left(Q^{*}\right)\right) \operatorname{det}^{1 / 2} G_{A B} d Q^{* 1} \wedge \ldots \wedge d Q^{* N_{\mathcal{P}}} .
$$

By taking an inversion of (23) we get the representation of standing under the sign of sum semigroups (the path integrals) in terms of the semigroups 
that are given on the manifold $\mathcal{P}$. As in [5], we will do it for the kernels of the corresponding local semigroups.

Provided that the analytical restrictions are fulfilled, the semigroup from the left-hand side of (23) ) can be presented as

$$
\psi_{t_{b}}\left(p_{a}, t_{a}\right)=\int G_{\mathcal{P}}\left(p_{b}, t_{b} ; p_{a}, t_{a}\right) \phi_{0}\left(p_{b}\right) d v_{\mathcal{P}}\left(p_{b}\right) .
$$

Using the partition of the unity subordinated to a local finite covering of the manifold $\mathcal{P}$ and having in mind that there is a local isomorphism of $P_{\Sigma}(\Sigma, \mathcal{G})$ with the trivial principal fiber bundle $\varphi_{\alpha_{b}}^{\Sigma}\left(\mathcal{U}_{\alpha_{b}}^{\Sigma}\right) \times \mathcal{G}$, by which a chart of the atlas of the manifold $\mathcal{P}$ is changed for the chart $\varphi_{\alpha_{b}}^{\Sigma}\left(\mathcal{U}_{\alpha_{b}}^{\Sigma}\right) \times \mathcal{G}$, we get the following expression for the right-hand side of (26):

$$
\int_{\varphi_{\alpha_{b}}^{\Sigma}} \tilde{\tilde{\mu}}_{\alpha_{b}}^{\Sigma}\left(x_{b}\right) G_{\mathcal{P}}\left(\alpha_{b}, F\left(Q_{b}^{*}, \theta_{b}\right), t_{b} ; \beta_{a}, F\left(Q_{a}^{*}, \theta_{a}\right), t_{a}\right) \tilde{\phi}_{0}\left(Q_{b}^{*}, \theta_{b}\right) d v\left(Q_{b}^{*}\right) d \mu\left(\theta_{b}\right),
$$

where $d v\left(Q^{*}\right)$ is the same volume measure as in (25) and $d \mu(\theta)=\operatorname{det} \bar{u}_{\beta}^{\alpha}(\theta) d \theta^{1} \ldots d \theta^{N_{\mathcal{G}}}$ is a Haar measure on a group $\mathcal{G}$.

Also, the right-hand side of (23) can be presented locally as

$$
\sum_{\alpha_{b}} \int_{\varphi_{\alpha_{b}}^{\Sigma}\left(\mathcal{U}_{\alpha_{b}}^{\Sigma}\right)} \tilde{\rho}_{\alpha_{b}}\left(x_{b}\right) \sum_{\lambda, p, q, q^{\prime}} G_{q^{\prime} p}^{\lambda}\left(\alpha_{b}, Q_{b}^{*}, t_{b} ; \beta_{a}, Q_{a}^{*}, t_{a}\right) c_{p q}^{\lambda}\left(Q_{b}^{*}\right) D_{q^{\prime} q}^{\lambda}\left(\theta_{a}\right) d v\left(Q_{b}^{*}\right) .
$$

Comparing (27) and (28) we find the relation between the local Green functions:

$$
\begin{aligned}
& \int_{\mathcal{G}} G_{\mathcal{P}}\left(\alpha_{b}, F\left(Q_{b}^{*}, \theta_{b}\right), t_{b} ; \beta_{a}, F\left(Q_{a}^{*}, \theta_{a}\right), t_{a}\right) D_{p q}^{\lambda}\left(\theta_{b}\right) d \mu\left(\theta_{b}\right)= \\
& \sum_{q^{\prime}} G_{q^{\prime} p}^{\lambda}\left(\alpha_{b}, Q_{b}^{*}, t_{b} ; \beta_{a}, Q_{a}^{*}, t_{a}\right) D_{q^{\prime} q}^{\lambda}\left(\theta_{a}\right),
\end{aligned}
$$

which, with account of the unimodularity of the group $\mathcal{G}$, can be easily reversed:

$$
G_{m n}^{\lambda}\left(\alpha_{b}, Q_{b}^{*}, t_{b} ; \beta_{a}, Q_{a}^{*}, t_{a}\right)=\int_{\mathcal{G}} G_{P}\left(\alpha_{b}, Q_{b}^{*}, \theta, t_{b} ; \beta_{a}, Q_{a}^{*}, e, t_{a}\right) D_{n m}^{\lambda}(\theta) d \mu(\theta) .
$$


In this formula $e$ corresponds to the unity element of the group $\mathcal{G}$ and

$$
G_{P}\left(\alpha_{b}, Q_{b}^{*}, \theta_{b}, t_{b} ; \beta_{a}, Q_{a}^{*}, \theta_{a}, t_{a}\right) \equiv G_{\mathcal{P}}\left(\alpha_{b}, F\left(Q_{b}^{*}, \theta_{b}\right), t_{b} ; \beta_{a}, F\left(Q_{a}^{*}, \theta_{a}\right), t_{a}\right) .
$$

Since in the paper we have confined ourselves by the case of the trivial principal fiber bundle, then the gluing these local Green functions to the global Green functions can be done with the transition coordinate functions defined for the charts of the manifolds.

Hence, the equality (29) can be extended from the local charts to the whole manifold and in result we obtain the relation between the Green function defined on the global manifolds:

$$
G_{m n}^{\lambda}\left(\pi_{\Sigma}\left(p_{b}\right), t_{b} ; \pi\left(p_{a}\right), t_{a}\right)=\int_{\mathcal{G}} G_{\mathcal{P}}\left(p_{b} \theta, t_{b} ; p_{a}, t_{a}\right) D_{n m}^{\lambda}(\theta) d \mu(\theta) .
$$

The path integral from the left-hand side of this equality can be written symbolically as

$$
\begin{aligned}
& G_{m n}^{\lambda}\left(\pi_{\Sigma}\left(p_{b}\right), t_{b} ; \pi_{\Sigma}\left(p_{a}\right), t_{a}\right)= \\
& \tilde{\mathrm{E}}_{\substack{\xi_{\Sigma}\left(t_{a}\right)=\pi_{\Sigma}\left(p_{a}\right) \\
\xi_{\Sigma}\left(t_{b}\right)=\pi_{\Sigma}\left(p_{b}\right)}}\left[(\overline{\exp })_{m n}^{\lambda}\left(\xi_{\Sigma}(t), t_{b}, t_{a}\right) \exp \left\{\frac{1}{\mu^{2} \kappa m} \int_{t_{a}}^{t_{b}} \tilde{V}\left(\xi_{\Sigma}(u)\right) d u\right\}\right] \\
& =\int_{\substack{\xi_{\Sigma}\left(t_{a}\right)=\pi_{\Sigma}\left(p_{a}\right) \\
\xi_{\Sigma}\left(t_{b}\right)=\pi_{\Sigma}\left(p_{b}\right)}}^{t^{2}} d \mu^{\xi_{\Sigma}} \exp \left\{\frac{1}{\mu^{2} \kappa m} \int_{t_{a}}^{t_{b}} \tilde{V}\left(\xi_{\Sigma}(u)\right) d u\right\} \\
& \times \overleftarrow{\exp } \int_{t_{a}}^{t_{b}}\left\{\frac { 1 } { 2 } \mu ^ { 2 } \kappa \left[\gamma^{\sigma \nu}\left(\xi_{\Sigma}(u)\right)\left(J_{\sigma}\right)_{m r}^{\lambda}\left(J_{\nu}\right)_{r n}^{\lambda}\right.\right. \\
& \left.-\left(G^{R S} \tilde{\Gamma}_{R S}^{B} \Lambda_{B}^{\beta}+G^{R P} \Lambda_{R}^{\sigma} \Lambda_{B}^{\beta} K_{P \sigma}^{B}-G^{C A} N_{C}^{M} \frac{\partial}{\partial Q^{* M}}\left(\Lambda_{A}^{\beta}\right)\right)\left(J_{\beta}\right)_{m n}^{\lambda}\right] d u \\
& \left.+\mu \sqrt{\kappa} \Lambda_{C}^{\beta}\left(J_{\beta}\right)_{m n}^{\lambda} \Pi_{K}^{C} \tilde{\mathfrak{X}}_{\bar{M}}^{K} d w^{\bar{M}}\right\} .
\end{aligned}
$$

The semigroup defined by this kernel acts in the space of the equivariant functions:

$$
\tilde{\psi}_{n}(p g)=D_{m n}^{\lambda}(g) \tilde{\psi}_{m}(p),
$$

that are isomorphic to the functions $\psi_{n}$ from the space of the sections $\Gamma\left(\Sigma, V^{*}\right)$ of the associated covector bundle:

$$
\tilde{\psi}_{n}\left(F\left(Q^{*}, e\right)\right)=\psi_{n}\left(Q^{*}\right)
$$


The method by which we have obtained the integral relation between $G_{m n}^{\lambda}$ and $G_{\mathcal{P}}$, can be regarded as the realization of the reduction procedure in the path integrals for the dynamical systems with a symmetry.

The reduction onto the zero-momentum level, i.e., when $\lambda=0$, establishes the the relation between the path integrals that are used for descriptions of a quantum motion of the scalar particle on an initial manifold $\mathcal{P}$ and on the the orbit space manifold $\mathcal{M}$.

In our case, in order to represent the motion on the orbit space, we have used an additional gauge surface $\xi_{\Sigma}$, on which the corresponding diffusion was given by the stochastic differential equations (14). In these equation there is an "extra" term, the drift $j_{I I}$, which is not directly related to the orbit space $\mathcal{M}$. Without this term we would have the stochastic process which could completely correspond to the diffusion on the orbit space.

In path integrals the transformation in which we change the stochastic process $\xi_{\Sigma}$ with the local stochastic differential equation (14) for the process $\tilde{\xi}_{\Sigma}$, with the stochastic differential equation

$$
d Q^{* A}(t)=\mu^{2} \kappa\left(-\frac{1}{2} G^{E M} N_{E}^{C} N_{M}^{B}{ }^{H} \Gamma_{C B}^{A}+j_{I}^{A}\right) d t+\mu \sqrt{\kappa} N_{C}^{A} \tilde{\mathfrak{X}}_{\bar{M}}^{C} d w^{\bar{M}},
$$

can be made with the help of the Girsanov transformation formula.

In our case, because of the presence of the projection operators in diffusion matrices of equations (14) and (32) we have the degenerate diffusion matrices. It restricts the application of the standard Girsanov formula.

But if we will remain in the frame of the predefined ambiguities, that originate from using of the projection operators, we can still derive the Girsanov formula. In our case it will be also based on the uniqueness (modulo the above ambiguity) of the solution of the parabolic differential equation with the operator given by the diagonal part of the operator (24) and on the application of the Itô differentiation formula for the composite function together with the account of the following formula:

$$
\left(G^{A B} N_{A}^{C} N_{B}^{D}\right)\left(\left(P_{\perp}\right)_{D}^{E} G_{E M}^{H}\left(P_{\perp}\right)_{L}^{M}\right)=\left(P_{\perp}\right)_{L}^{C} .
$$

In result, the Radon-Nicodim derivative of the measure $\mu^{\xi_{\Sigma}}$ with respect to the measure $\mu^{\tilde{\xi}_{\Sigma}}$ will be as follows:

$$
\begin{aligned}
& \frac{d \mu^{\xi_{\Sigma}}}{d \mu^{\tilde{\xi}_{\Sigma}}}\left(\tilde{\xi}_{\Sigma}(t)\right)=\exp \int_{t_{a}}^{t}\left[-\frac{1}{2} \mu^{2} \kappa\left(\left(P_{\perp}\right)_{A}^{L} G_{L K}^{H}\left(P_{\perp}\right)_{E}^{K}\right) j_{I I}^{A} j_{I I}^{E} d t\right. \\
& \left.+\mu \sqrt{\kappa} G_{L K}^{H}\left(P_{\perp}\right)_{A}^{L} j_{I I}^{A} \tilde{\mathfrak{X}}_{\bar{M}}^{K} d w^{\bar{M}}\right] .
\end{aligned}
$$


Performing such a change of the integration variables in that path integral which is obtained as a result of the reduction to $\lambda=0$ momentum level, we get the following integral relation:

$$
G_{\Sigma}\left(Q_{b}^{*}, t_{b} ; Q_{a}^{*}, t_{a}\right)=\int_{\mathcal{G}} G_{\mathcal{P}}\left(p_{b} \theta, t_{b} ; p_{a}, t_{a}\right) d \mu(\theta),
$$

where the kernel $G_{\Sigma}$ is presented by the path integral

$$
\begin{aligned}
& G_{\Sigma}\left(Q_{b}^{*}, t_{b} ; Q_{a}^{*}, t_{a}\right)=\int_{\substack{\tilde{\xi}_{\Sigma}\left(t_{a}\right)=Q_{a}^{*} \\
\tilde{\xi}_{\Sigma}\left(t_{b}\right)=Q_{b}^{*}}} d \mu^{\tilde{\xi}_{\Sigma}} \exp \left\{\frac{1}{\mu^{2} \kappa m} \int_{t_{a}}^{t_{b}} V\left(\tilde{\xi}_{\Sigma}(u)\right) d u\right\} \\
& \times \exp \int_{t_{a}}^{t_{b}}\left\{-\frac{1}{8} \mu^{2} \kappa G^{A B} N_{A}^{D} N_{B}^{L}\left[\gamma^{\alpha \beta} G_{D C}\left(\tilde{\nabla}_{K_{\alpha}} K_{\beta}\right)^{C}\right]\right. \\
& \left.\times\left[\gamma^{\mu \nu} G_{L E}\left(\tilde{\nabla}_{K_{\mu}} K_{\nu}\right)^{E}\right] d t+\frac{1}{2} \mu \sqrt{\kappa} N_{P}^{D}\left[\gamma^{\alpha \beta} G_{C D}\left(\tilde{\nabla}_{K_{\alpha}} K_{\beta}\right)^{C}\right] \tilde{\mathfrak{X}}_{\bar{M}}^{P} d w^{\bar{M}}\right\} \\
& \left(Q^{*}=\pi_{\Sigma}(p)\right) .
\end{aligned}
$$

The semigroup determined by this path integral acts in the space of the scalar functions given on $\Sigma$.

Remarks, that there is a difference between the formula obtained here and an analogous formula from [5. In the present formula the reduction Jacobian has an additional stochastic integral. It is possible to get rid of this stochastic integral with the help of the corresponding Itô identity. As it needs an additional investigation we does not make this transformation in the present paper.

\section{Conclusion}

From our path integral transformation it follows that the path integral measure is not invariant under the reduction (the formulas (30) and (31)).

The obtained reduction Jacobian reveals an interesting geometrical structure. Namely, it is related with the mean curvature vector of the orbit over the point belonging to the base space in the principal fiber bundle. After replacement of the variables in the path integral this mean curvature together with the mean curvature of the orbit space adds to the standard drift term of the stochastic differential equation (14). 
We may suppose that the sum of two mean curvature comes from the mean curvature of the manifold $\mathcal{P}$ provided that it is considered as being embedded in some manifold with a bigger dimension.

Acknowledgments. I thank to A.V.Razumov for the discussion of various geometrical problems, V.O.Soloviev and V.I.Borodulin for valuable advises and help.

\section{Appendix A}

\section{Stochastic differential equation on a submani- fold}

Let the manifold $\mathcal{M}$ be embedded into the smooth (compact) finite dimensional Riemannian manifold with a metric $G_{A B}(Q)$. We assume that this embedding is locally given by the equations $Q^{A}=Q^{A}\left(x^{i}\right)$, where $\left\{Q^{A}\right\}$ is a coordinate system on the external manifold and $\left\{x^{i}\right\}-$ on $\mathcal{M}$. Then, on $\mathcal{M}$ we have an induced metric: $h_{i j}(x)=Q_{i}^{A}(x) Q_{j}^{B}(x) G_{A B}(Q(x))$.

The stochastic process $\xi(t)=\left\{x^{i}(t)\right\}$, with the differential generator $1 / 2 \triangle_{\mathcal{M}}\left(\triangle_{\mathcal{M}}\right.$ is a Laplace - Beltrami operator on $\left.\mathcal{M}\right)$ can be determined by the solution of the stochastic differential equation with the following local representation:

$$
\begin{aligned}
& d x^{k}(t)=-\frac{1}{2} h^{i j}(x(t)) \Gamma_{i j}^{k}(x(t)) d t+X_{\bar{m}}^{k}(x(t)) d w^{\bar{m}}(t), \\
& \left(\sum_{\bar{m}} X_{\bar{m}}^{k} X_{\bar{m}}^{l}=h^{k l}\right) .
\end{aligned}
$$

Now we will define the same stochastic process, but we will make use the variables $Q^{A}$ that are related with the external manifold. We assume that the stochastic differential equation which describes the stochastic process on a submanifold can be written as

$$
d Q^{A}(t)=a^{A} d t+\tilde{\mathfrak{X}}_{\bar{M}}^{A} d w^{\bar{M}}(t),
$$

where $a^{A}$ and $\tilde{\mathfrak{X}}_{\bar{M}}^{A}(t)$ are some (and not defined yet) functions of $Q(t)$. Also, we require that at the initial moment of time the process $Q^{A}(t)$ be on the submanifold $\mathcal{M}$.

In order to find the explicit expressions for the coefficients of equation (A.2) we will apply the Itô differentiation formula to the function $Q^{A}=$ 
$Q^{A}\left(x^{i}(t)\right)$. As for the stochastic variables $x^{i}(t)$, we will assume that they satisfy the equation (A.1).

Then, comparing the result of such a differentiation with the expression in the right-hand side of (A.2), we find that the coefficient $a^{A}$ is equal to

$$
a^{A}=-\frac{1}{2} Q_{i}^{A}(x(t)) h^{k l}(x(t)) \Gamma_{k l}^{i}(x(t))+\frac{1}{2} Q_{i j}^{A}(x(t)) h^{i j}(x(t)) .
$$

But

$$
\begin{aligned}
& h^{k l}(x) \Gamma_{k l}^{i}(x)=G_{A B}(Q(x))\left(Q_{k l}^{A}(x)+\right. \\
& \left.\quad+\Gamma_{C D}^{A}(Q(x)) Q_{k}^{C}(x) Q_{l}^{D}(x)\right) h^{i m}(x) Q_{m}^{B}(x) h^{k l}(x)
\end{aligned}
$$

(see, for example, 22]). Taking this into account and using the projection onto the tangent space to the manifold $\mathcal{M}$ :

$$
N_{B}^{C}(Q(x))=G_{B A}(Q(x)) Q_{i}^{A}(x) h^{i j}(x) Q_{j}^{C}(x),
$$

we can transform (A.3) to another form

$$
a^{A}=-\frac{1}{2} N_{P}^{A} h^{i j} Q_{i}^{C} Q_{j}^{D} \Gamma_{C D}^{P}-\frac{1}{2} N_{P}^{A} Q_{k l}^{P} h^{k l}+\frac{1}{2} Q_{k l}^{A} h^{k l} .
$$

Since the components of the mean curvature vector of the submanifold is given by

$$
\begin{aligned}
j^{D} & =\frac{1}{2}\left(\delta_{B}^{D}-N_{B}^{D}\right) h^{i j}\left[\nabla_{Q_{i}^{P} \frac{\partial}{\partial Q^{P}}}\left(Q_{j}^{L} \frac{\partial}{\partial Q^{L}}\right)\right]^{B} \\
& =\frac{1}{2} h^{i j}\left(Q_{i}^{A} Q_{j}^{B} \Gamma_{A B}^{D}+Q_{i j}^{D}-N_{C}^{D} Q_{i}^{A} Q_{j}^{B} \Gamma_{A B}^{C}-N_{C}^{D} Q_{i j}^{C}\right),
\end{aligned}
$$

we can rewrite A.5 follows:

$$
a^{A}(Q(x))=-\frac{1}{2} G^{E M}(Q(x)) N_{E}^{C}(Q(x)) N_{M}^{B}(Q(x)) \Gamma_{C B}^{A}(Q(x))+j^{A} .
$$

where $j^{A}$ is, in fact, the function given on a submanifold, i.e., $j^{A} \equiv j^{A}(Q(x))$. This follows from the fact that the mean curvature can be also defined without using an explicit coordinate expression (for example, by the Weingarten map).

Before proceeding to the determination of the diffusion coefficient $\tilde{\mathfrak{X}}_{\bar{M}}^{A}(t)$ we notice, that the difussion coefficients of the equations (A.1) and (A.2) are defined only up to the orthogonal transformations. 
From the equality

$$
\tilde{\mathfrak{X}}_{\bar{M}}^{A} d w^{\bar{M}}=Q_{i}^{A} X_{\bar{m}}^{i} d w^{\bar{m}},
$$

which can be derived from (A.2) in result of the application of the Itô differentiation formula, it follows that

$$
\sum_{\bar{M}} \tilde{\mathfrak{X}}_{\bar{M}}^{A} \tilde{\mathfrak{X}}_{\bar{M}}^{B}=\sum_{\bar{m}} Q_{i}^{A} X_{\bar{m}}^{i} Q_{j}^{B} X_{\bar{m}}^{j}=h^{i j} Q_{i}^{A} Q_{j}^{B}=G^{C D} N_{C}^{A} N_{D}^{B}
$$

These equations define $\tilde{\mathfrak{X}}_{\bar{M}}^{A}$ :

$$
\tilde{\mathfrak{X}}_{\bar{M}}^{A}=N_{C}^{A} \mathfrak{X}_{\bar{M}}^{C}, \quad\left(\sum_{\bar{M}} \mathfrak{X}_{\bar{M}}^{D} \mathfrak{X}_{\bar{M}}^{C}=G^{C D}\right) .
$$

At last, redefining the coordinates $Q^{A}(x(t))$ of the stochastic process for new coordinates $Q^{A}(t)$ (together with the requirement, that at the initial moment of time a new process was also given on a submanifold ), we get the following local stochastic differential equation for the components of the stochastic process on a submanifold $\mathcal{M}$ :

$$
d Q^{A}(t)=\left(-\frac{1}{2} G^{E M} N_{E}^{C} N_{M}^{D} \Gamma_{C D}^{A}+j^{A}\right) d t+N_{C}^{A} \mathfrak{X}_{\bar{M}}^{C} d w^{\bar{M}}
$$

where all the functions in right-hand side of this equation depend on $Q^{A}(t)$.

\section{References}

[1] L. D. Faddeev, Teor. i Mat. Fyz. 1 (1969) 3 (in Russian);

L. D. Faddeev, V. N. Popov, Phys. Lett. 25B (1967) 30.

[2] N. P. Landsman, N. Linden, Nucl. Phys. B365 (1991) 121;

S. Tanimura, I. Tsutsui, Mod. Phys. Lett A34 (1995) 2607;

D. McMullan, I.Tsutsui, Ann. Phys. 237 (1995) 269.

[3] G. Kunstatter, Class. Quant.Grav. 9 (1992) 1466.

[4] S. N. Storchak, J. Phys. A: Math. Gen. 34 (2001) 9329.

[5] S. N. Storchak, Bogolubov transformation in path integrals on manifold with a group action. IHEP Preprint 98-1, Protvino, 1998. 
[6] P. K. Mitter, C. M. Viallet, Comm. Math. Phys. 79 (1981) 457.

[7] H. Hüffel, G. Kelnhofer, Ann. of Phys. 266 (1998) 417; Ann. of Phys. 270 (1998) 231.

[8] Z. Jaskolski, Comm. Math. Phys. 111 (1987) 439.

[9] N. K. Falck, A. C. Hirshfeld, Ann. Phys. 144 (1982) 34;

K. Gavedzki, Phys. Rev. D26 (1982) 3593.

[10] P. Ellicott, G. Kunstatter and D. J. Toms, Mod. Phys. Lett. A4 n.24 (1989) 2397.

[11] Ya. I. Belopolskaya, Yu. L. Dalecky, Usp. Mat. Nauk 37 n.3 (1982) 95 (in Russian);

Yu. L. Dalecky, Usp. Mat. Nauk 38 n.3 (1983) 87 (in Russian);

Ya. I. Belopolskaya and Yu. L. Dalecky, Stochastic equations and differential geometry (Mathematics and Its Applications: Soviet Series, 30. Dordrecht etc.: (Kluwer Academic Publishers, 1990).

[12] R. Abraham, J. E. Marsden, Foundation of Mechanics, 2nd Ed. (Addison-Wesley Redwood City, 1985).

[13] O. A. Khrustalev,A. V. Razumov, A. Yu. Taranov, Nucl. Phys. B172 (1980) 44;

A. V. Razumov, A. Yu. Taranov, Teor. i Mat. Fyz. 52 (1982) 34 (in Russian);

IHEP Preprint 82-41, Serpukhov, 1982;

A. V. Razumov, Bogolubov Transformation and Quantum Theory of Constrained Systems, Dissertation (Protvino, 1991) (in Russian).

[14] E. P. Solodovnikova, A. N. Tavkhelidze, O. A. Khrustalev Teor. i Mat. Fyz. 10 (1972) 162; Teor. i Mat. Fyz. 11 (1972) 372; Teor. i Mat. Fyz. 12 (1972) 164.

[15] M. Creutz, I. J. Muzinich and Th. N. Tudron, Phys. Rev. D19, n.2 (1979) 531.

[16] M. S. Plyushchay, A. V. Razumov, Int. J. Mod. Phys. A11, n.8 (1996) 1427. 
[17] J. T. Lewis, Bull. London Math. Soc. 18 (1986) 616.

[18] R. S. Lipster and A. N. Shiryayev, Statistics of Random Processes, Vols. I and II (Springer-Verlag: Berlin, Heidelberg, New York, 1977).

[19] V. S. Pugachev and I. N. Sinitsyn, Stochastic differential systems, 2nd Edition (Moscow, Nauka, 1990) (in Russian).

[20] Yu. L. Dalecky, N. I. Teterina, Usp. Mat. Nauk 27, n.2 (1972) 167 (in Russian);

Yu. L. Dalecky, Usp. Mat. Nauk 38 n.2 (1975) 209 (in Russian);

D. W. Stroock, Com. Pure Appl. Math. 23 (1970) 447.

[21] N. Ikeda and S. Watanabe, Stochastic differential equations and diffusion processes (North-Holland, Amsterdam, 1981). S. N. Storchak, Teor. $i$ Mat. Fyz. 75 (1988) 610.

[22] D. E. Betounes, Phys. Rev. D33, n.12 (1980) 3634;

Bang-yen Chen, Geometry of Submanifold (Marsel Deccer, N.Y., 1973). 